\title{
Ultrastructure of the vitreoretinal interface following plasmin assisted vitrectomy
}

\author{
A Gandorfer, E Putz, U Welge-Lüßen, M Grüterich, M Ulbig, A Kampik
}

\begin{abstract}
Aims-To investigate the ultrastructure of the vitreoretinal interface following plasmin induced posterior vitreous detachment. Methods-Plasmin ( 1 or $2 \mathrm{U} / 0.1 \mathrm{ml}$ ) was injected into the vitreous cavity of 24 eyes of freshly slaughtered pigs. The 24 fellow eyes received calcium-free and magnesium-free PBS and served as a control. After incubation at $37^{\circ} \mathrm{C}$ for 30 and 60 minutes, the globes were placed in fixative and hemisected. Specimens for light, scanning, and transmission electron microscopy were obtained from the posterior pole, the equator, and the vitreous base using a corneal trephine.

Results-All plasmin treated eyes showed posterior vitreous detachment. However, the inner limiting membrane (ILM) was covered by remnants of cortical vitreous at the posterior pole and at the equator. There was a direct correlation between the concentration and exposure times of plasmin and the degree of vitreoretinal separation. Eyes exposed to $1 \mathrm{U}$ plasmin for $\mathbf{3 0}$ minutes had a dense network of residual collagen fibrils while those exposed to $1 \mathrm{U}$ plasmin for 60 minutes had only sparse collagen fibrils covering the ILM. Eyes treated with $2 \mathrm{U}$ plasmin for 60 minutes had a smooth retinal surface, consistent with a bare ILM. At the vitreous base there was no vitreoretinal separation. In all control eyes the vitreous cortex was completely attached to the retina. There was no evidence of retinal damage in any plasmin treated eye.

Conclusion-Plasmin induces a cleavage between the vitreous cortex and the ILM without morphological changes to the retina. In contrast with previous reports, plasmin produces a smooth retinal surface and additional surgery is not required in this experimental setting. The degree of vitreoretinal separation depends on the concentration and length of exposure to plasmin.

(Br f Ophthalmol 2001;85:6-10)
\end{abstract}

Separation of the posterior hyaloid from the inner limiting membrane (ILM), a condition clinically known as posterior vitreous detachment (PVD), has an impact on the course of various vitreoretinal disorders including vitreo- macular traction syndrome, macular hole formation, retinal detachment, diabetic retinopathy, and retinal vein occlusion. ${ }^{1-16}$

Relief of traction forces from the retina is a primary aim of vitreoretinal surgery. ${ }^{17-19}$ Despite improved surgical technique and skills, complete mechanical removal of the vitreous cortex from the retinal surface remains difficult. $^{20-22}$ Surgical manipulation may cause retinal breaks and haemorrhage, particularly in children and young adults. Moreover, even in the presence of PVD, remnants of the posterior hyaloid provide a scaffold for subsequent cellular proliferation and fibrocellular contraction, resulting in epiretinal membrane formation and proliferative vitreoretinopathy. ${ }^{23}{ }^{24}$ Cleaving the vitreoretinal interface selectively and completely by biochemical means is therefore a desirable adjunct or alternative to vitrectomy. ${ }^{25}$

Plasmin, a serine protease mediating the fibrinolytic process, hydrolyses a number of glycoproteins including laminin and fibronectin, both of which are present at the vitreoretinal interface and support attachment of the vitreous cortex to the ILM. ${ }^{26-30}$ It has been reported previously that an intravitreal injection of plasmin facilitates the formation of PVD in combination with vitrectomy or intravitreal injection of hexafluoride gas. ${ }^{31-33}$

The morphology of the vitreoretinal interface of plasmin treated eyes has not been studied and published in detail previously. There are no reliable data on the success of proteolytic vitreoretinal separation or the dose of plasmin needed and exposure times required. We therefore exposed eyes from freshly slaughtered pigs to different concentrations and exposure times of plasmin and investigated the ultrastructure of the vitreoretinal interface at the posterior pole, the equator, and the vitreous base by light and electron microscopy following treatment.

\section{Materials and methods}

PLASMIN INJECTION

Stock lyophilised plasmin powder (EC 3.4.21.7, Sigma-Aldrich, Germany) from porcine blood containing a proteolytic activity of $5 \mathrm{U} / \mathrm{mg}$ was prepared in calcium-free and magnesium-free phosphate buffered saline (PBS), diluted to a final concentration of $1 \mathrm{U}$ and $2 \mathrm{U} / 0.1 \mathrm{ml}$, and stored at $-20^{\circ} \mathrm{C}$ until further use. 
Table 1 Scanning electron microscopy of the vitreoretinal interface

\begin{tabular}{|c|c|c|c|c|}
\hline \multirow{2}{*}{$\begin{array}{l}\text { Concentration and } \\
\text { duration of exposure }\end{array}$} & \multirow[b]{2}{*}{ No of eyes } & \multicolumn{3}{|c|}{ Remnants of vitreous cortex } \\
\hline & & Posterior pole & Equator & Vitreous base \\
\hline \multirow[t]{5}{*}{$1 \mathrm{U}$ plasmin, 30 minutes } & \multirow[t]{5}{*}{5} & ++ & ++ & +++ \\
\hline & & ++ & ++ & +++ \\
\hline & & ++ & +++ & +++ \\
\hline & & ++ & ++ & +++ \\
\hline & & ++ & ++ & +++ \\
\hline \multirow[t]{5}{*}{$1 \mathrm{U}$ plasmin, 60 minutes } & \multirow[t]{5}{*}{5} & + & + & +++ \\
\hline & & + & ++ & +++ \\
\hline & & + & + & +++ \\
\hline & & + & + & +++ \\
\hline & & ++ & ++ & +++ \\
\hline \multirow[t]{5}{*}{$2 \mathrm{U}$ plasmin, 30 minutes } & \multirow[t]{5}{*}{5} & + & + & +++ \\
\hline & & + & + & +++ \\
\hline & & + & + & +++ \\
\hline & & - & + & +++ \\
\hline & & + & + & +++ \\
\hline \multirow[t]{5}{*}{$2 \mathrm{U}$ plasmin, 60 minutes } & \multirow[t]{5}{*}{5} & - & + & +++ \\
\hline & & - & + & +++ \\
\hline & & - & + & +++ \\
\hline & & - & + & +++ \\
\hline & & - & + & +++ \\
\hline Plasmin treated eyes & & & & \\
\hline without incubation & 4 & +++ & +++ & +++ \\
\hline Control fellow eyes & 24 & +++ & +++ & +++ \\
\hline
\end{tabular}

$+++=$ No vitreoretinal separation, vitreous cortex attached to the inner limiting membrane (ILM); ++ = vitreoretinal separation but continuous network of collagen fibrils covering the ILM; $+=$ sparse collagen fibrils covering the ILM; - = bare ILM.

Fresh adult domestic pig eyes were harvested from the local slaughterhouse and carried on ice to the laboratory within 1 hour. The eyes were cleaned of extraneous tissue and incubated at $37^{\circ} \mathrm{C}$ for 15 minutes. Within 90 minutes of being slaughtered plasmin $(1 \mathrm{U} /$ $0.1 \mathrm{ml}$ ) was injected via the pars plana into the mid vitreous cavity of 12 eyes; 12 fellow eyes received $0.1 \mathrm{ml}$ of calcium-free and magnesium-free PBS and served as a control. Five plasmin treated eyes and their control fellow eyes were incubated at $37^{\circ} \mathrm{C}$ for 30 minutes, and five of each were incubated for 60 minutes. As a control, two eyes received an
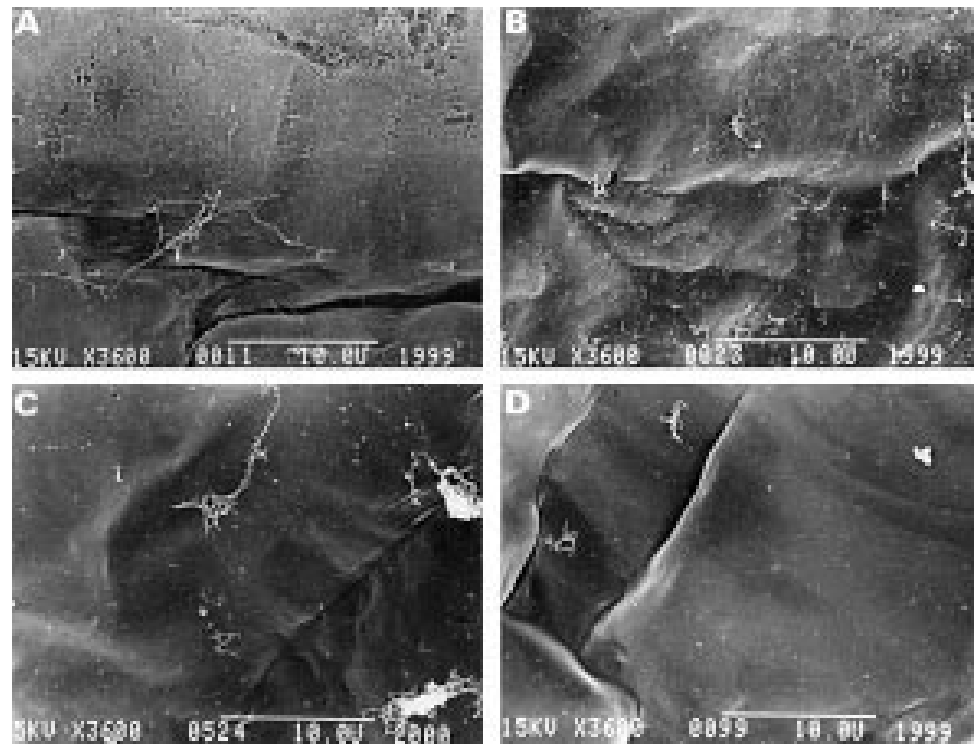

Figure 1 Scanning electron micrographs of the posterior pole of plasmin treated eyes showing remnants of cortical vitreous. There was a direct correlation between the degree of vitreoretinal separation and the concentration of plasmin and duration of exposure. (A) Eyes which had received $1 \mathrm{U}$ plasmin for 30 minutes showed a continuous network of persistent collagen fibrils covering major parts of the ILM. (B) In eyes which had received 1 U plasmin for 60 minutes only sparse collagen fibrils were delineated on the ILM. $(C)$ Eyes which were incubated with $2 U$ plasmin for 30 minutes had sparse collagen fibrils covering the ILM while those which had received $2 U$ plasmin for 60 minutes (D) had a smooth retinal surface consistent with a bare ILM. Magnification $\times 3600 ; 1$ bar $=10 \mu \mathrm{m}$. intravitreal injection of plasmin (the fellow eyes received PBS), but were not incubated. In another 12 eyes, $0.1 \mathrm{ml}$ of plasmin in a concentration of $2 \mathrm{U} / 0.1 \mathrm{ml}$ was used; the 12 fellow eyes received $0.1 \mathrm{ml}$ PBS.

\section{HISTOLOGICAL EXAMINATION}

After incubation, or immediately following intravitreal injection of plasmin or PBS in eyes not incubated, four sclerotomies were made $3 \mathrm{~mm}$ posterior to the limbus to ensure rapid penetration of the fixative. The globes were then fixed in a solution of $4 \%$ formaldehyde in PBS at $4^{\circ} \mathrm{C}$ for 24 hours. The globes were opened into two calottes and a central ring following the anteroposterior axis. The central ring was processed for light microscopy by dehydration in ethanol, embedded in paraffin, and cut in sections of $7 \mu \mathrm{m}$ thickness. The sections were stained with haematoxylin/eosin, periodic acid for PAS reaction, and colloidal iron stain for acid mucopolysaccharides.

Retinal specimens for scanning and transmission electron microscopy were obtained from the posterior pole, the equator, and the vitreous base using a corneal trephine of $4 \mathrm{~mm}$ diameter. Retinal discs for scanning electron microscopy (SEM) were postfixed in osmium tetroxide 2\% (Dalton's fixative), dehydrated in ethanol, dried to the critical point, sputter coated in gold, and photographed using an ISM-35 CF electron microscope (Jeol, Tokyo, Japan).

Specimens for transmission electron microscopy (TEM) were postfixed in Dalton's fixative, dehydrated, and embedded in Epon. Semithin sections were stained with $2 \%$ toluidine blue. Ultrathin sections were contrasted with uranyl acetate and lead citrate and analysed using a Zeiss EM 9 electron microscope (Zeiss, Jena, Germany).

Electron microscopic photographs were evaluated by two observers independently. Each observer evaluated the degree of vitreoretinal separation using a standardised grading system (Table 1 ).

\section{Results}

LIGHT MICROSCOPY

The retinal morphology of the porcine eye resembles the human eye. ${ }^{34}$ There are distinct cellular layers and a continuous ILM covering the inner surface of the retina. In our series light microscopy showed normal retinal morphology and cellular anatomy. The cellular layers of the retina were clearly demarcated and showed no abnormalities compared with porcine eyes previously described in the literature. ${ }^{35}$ In all eyes the ILM was present as a continuous membranous structure.

However, there are some clear differences between the porcine and human retina. Pigs, as well as cats and dogs, do not have a central retinal artery. In the human retina the central retinal artery splits into four branches forming the vascular arcades on the temporal and nasal side of the optic disc. By contrast, in the porcine eye it splits into only three branches. ${ }^{36} 37$ 

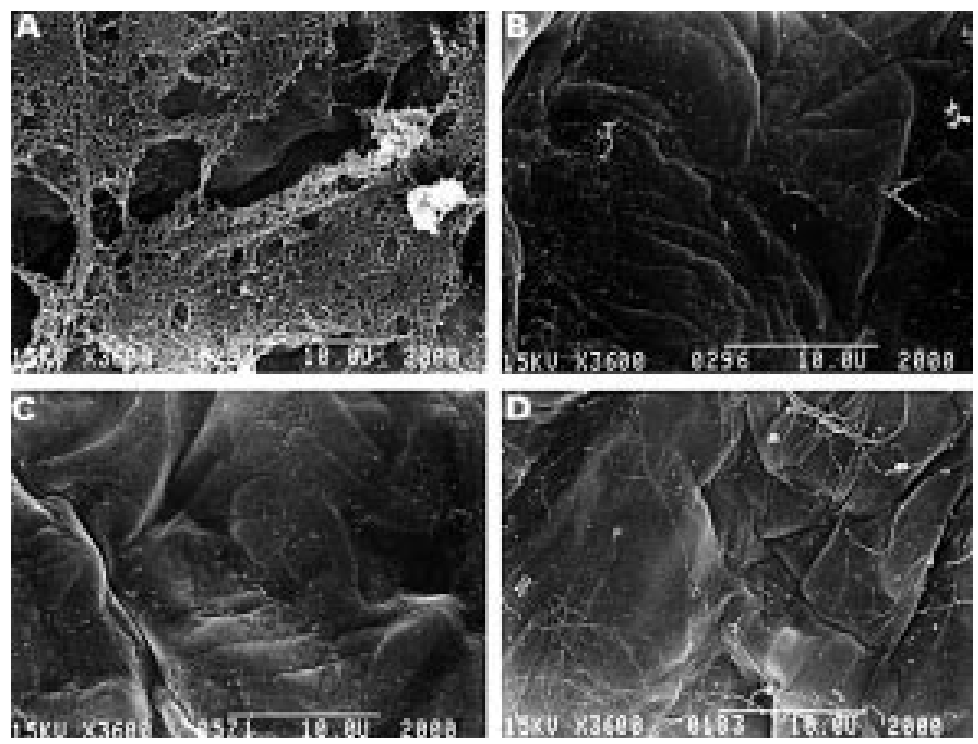

Figure 2 Scanning electron micrographs of the equator of plasmin treated eyes showing remnants of cortical vitreous. (A) Eyes which had received 1 U plasmin for 30 minutes had a continuous network of collagen fibrils covering the ILM while those which received $1 \mathrm{U}$ plasmin for 60 minutes (B), 2 U plasmin for 30 minutes $(C)$, or 2 U plasmin for 60 minutes (D) had only sparse collagen fibrils covering the ILM. Magnification $\times 3600 ; 1$ bar $=10 \mu \mathrm{m}$.
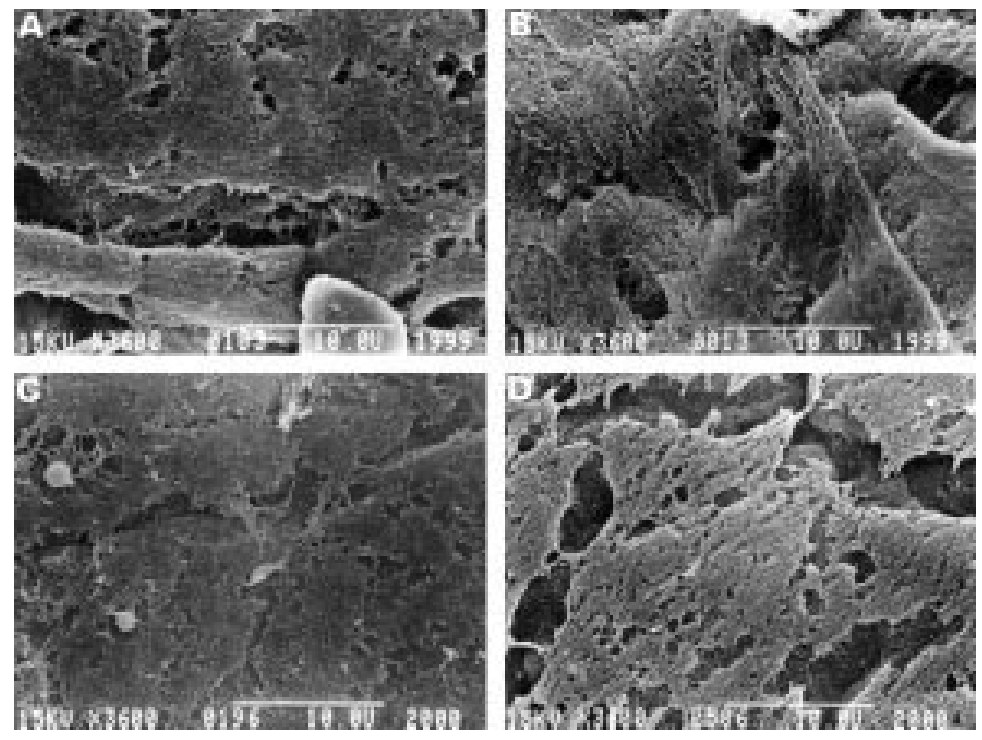

Figure 3 All controls had an attached cortical vitreous at the posterior pole $(A)$, at the equator (B), and at the vitreous base $(C)$. At the vitreous base there was no vitreoretinal separation in plasmin treated eyes (D). Magnification $\times 3600 ; 1 \mathrm{bar}=10 \mu \mathrm{m}$.

Little is known about the ultrastructure of the vitreoretinal interface in porcine eyes. In our series all eyes which had received an intravitreal injection of PBS and served as a control, and the four plasmin treated eyes which were not incubated, had an attached vitreous. This is in accordance with previous studies which have reported a strong vitreoretinal attachment in porcine eyes. ${ }^{38}$

All plasmin treated eyes which had been incubated had PVD. At the vitreous base the vitreous cortex was attached to the retina in both plasmin treated eyes and in controls.

SCANNING ELECTRON MICROSCOPY

All plasmin treated eyes had PVD after incubation. However, remnants of cortical vitreous were present at the posterior pole and at the equator. There was a direct correlation between both the concentration of plasmin used and the length of exposure and the degree of vitreoretinal separation (Table 1 ).

\section{Posterior pole}

Eyes which were treated with $0.1 \mathrm{ml}$ plasmin at a concentration of $1 \mathrm{U}$ for 30 minutes showed a continuous network of persistent collagen fibrils covering major parts of the ILM (Fig 1A). Longer exposure times of plasmin reduced the presence of remnants of cortical vitreous. In eyes treated with $1 \mathrm{U}$ plasmin for 60 minutes and those given $2 \mathrm{U}$ plasmin for 30 minutes only sparse collagen fibrils were delineated on the ILM (Fig $1 \mathrm{~B}$ and C). Eyes given $0.1 \mathrm{ml}$ plasmin at a concentration of $2 \mathrm{U}$ for 60 minutes had a smooth retinal surface, consistent with bare ILM (Fig 1D).

\section{Equator}

In eyes which had been incubated with $1 \mathrm{U}$ plasmin for 30 minutes there was a continuous network of persistent collagen fibrils covering the ILM (Fig 2A). Eyes injected with $1 \mathrm{U}$ plasmin for 60 minutes and $2 \mathrm{U}$ plasmin for 30 and 60 minutes had only sparse collagen fibrils on the ILM (Fig 2B, C, D).

\section{Vitreous base}

At the vitreous base all plasmin treated and control eyes had an attached cortical vitreous. In PBS treated controls and plasmin treated eyes with no previous incubation the vitreous cortex was attached to the ILM at the posterior pole, the equator, and the vitreous base (Fig 3).

\section{Variation within groups and interobserver error}

There was only a small amount of variation in the degree of vitreoretinal separation within groups treated with different concentrations and exposure times of plasmin (Table 1).

All histological evaluations were graded by two observers independently. The interobserver error was 4/144. All four disagreements concerned eyes treated with $1 \mathrm{U}$ plasmin for 30 and 60 minutes. In these questionable cases the vitreoretinal separation was graded as a "continuous network of collagen fibrils" and not as "sparse collagen fibrils covering the ILM".

\section{TRANSMISSION ELECTRON MICROSCOPY}

Transmission electron micrographs of controls showed persistent vitreous attachment with collagen fibres condensed over the retinal surface. In plasmin treated eyes residual collagen fibrils were observed adjacent to the ILM in some areas. Eyes injected with $0.1 \mathrm{ml}$ plasmin in a concentration of $2 \mathrm{U}$ for 30 and 60 minutes showed no evidence of vitreous collagen on the retinal surface.

The morphology of the ILM and the cellular anatomy of the retina were unchanged. In particular, the ILM was not affected after plasmin treatment. There was no difference in the lamina densa, the lamina lucida, and the adjacent Mueller cells between plasmin treated and control eyes (Fig 4). 

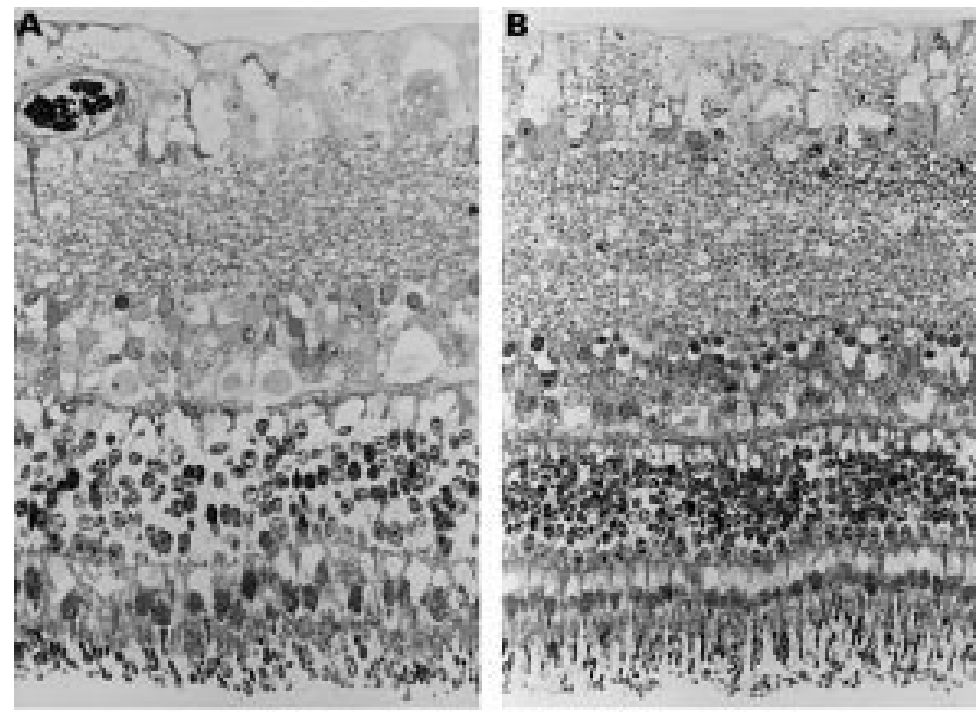

c

D

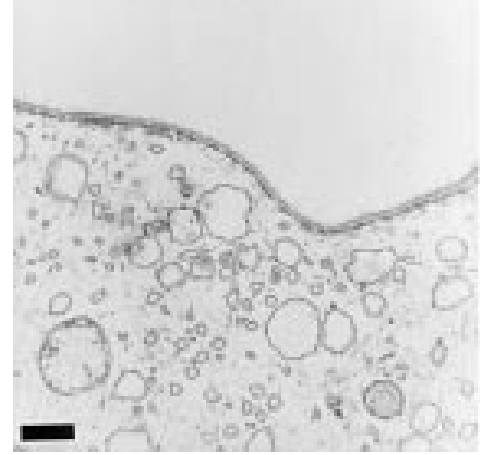

Figure 4 The retina was not affected by plasmin treatment. Semithin sections revealed normal morphology and cellular anatomy of the retina in $(A)$ plasmin treated eyes and $(B)$ controls (toluidine blue stain; magnification $\times 250$ ). Transmission electron microscopy

showed no difference in the ultrastructure of the ILM between (C) plasmin treated eyes and (D) controls. Magnification $\times 15858 ; 1$ bar $=6 \mu \mathrm{m}$. Note bare ILM in plasmin treated eyes $(A$ and $C)$ and residual vitreous cortex in control eyes $(B$ and $D)$.

\section{Discussion}

It has previously been reported that plasmin in combination with vitrectomy or intravitreal gas injection facilitates the cleavage between the vitreous cortex and the ILM. ${ }^{31-33}$ However, the ultrastructure of the vitreoretinal interface and the degree of vitreoretinal separation following different concentrations and exposure times of plasmin have not previously been reported in detail.

This study has shown that plasmin alone produces a cleavage at the vitreoretinal interface of porcine eyes in a reproducible manner. Besides intravitreal injection of plasmin, no additional surgical technique was required to induce PVD. Moreover, there was a direct correlation between the concentration and exposure time of plasmin and the degree of vitreoretinal separation at the posterior pole and at the equator.

There was no difference between plasmin treated eyes which had not been incubated and controls, indicating an immediate inactivation of plasmin by the fixative. The length of exposure to the proteolytic effect of plasmin was therefore limited to 30 and 60 minutes, providing reliable and comparable data as a basis for further studies.
Despite the presence of PVD in all plasmin treated eyes after incubation, there was a dense and continuous network of residual collagen fibrils covering the ILM in eyes which had received $1 \mathrm{U}$ plasmin for 30 minutes. Extending the exposure time to 60 minutes reduced the remnants of cortical vitreous significantly. The most complete vitreoretinal separation consistent with a bare ILM was achieved after 60 minutes of plasmin activity at a concentration of $2 \mathrm{U}$.

In contrast with previous reports, we were able to induce PVD using plasmin and no additional surgery. In rabbit eyes treated with plasmin Verstraeten et al found only eyes which underwent additional vitrectomy had a smooth retinal surface. ${ }^{32}$ Hikichi and coworkers observed complete vitreoretinal separation only in plasmin treated eyes which were additionally treated with $0.5 \mathrm{ml}$ hexafluoride gas. ${ }^{31}$ Verstraeten et al achieved best results of vitrectomy using $1 \mathrm{U}$ plasmin and an exposure time of 60 minutes. ${ }^{32}$ However, both Verstraeten and Hikichi used human plasmin in a rabbit model. To our knowledge there are no data available on the activity of plasmin of different species, but we assume a change in the proteolytic activity of plasmin when used in another species. In our study, in which porcine plasmin was used in porcine eyes, complete cleavage between the ILM and the vitreous cortex was achieved without vitrectomy.

Eyes from freshly slaughtered animals are not affected by a breakdown in the bloodretinal barrier and are thus an ideal model for studying the proteolytic effect of plasmin at the vitreoretinal interface. In previous in vivo studies breakdown of the blood-retinal barrier in rabbit eyes may have interfered with the proteolytic activity of plasmin. This may have contributed to the less than satisfactory vitreoretinal separation in previous series when plasmin was used without additional surgery.

Besides the proteolytic activity of plasmin against fibrin, laminin, and fibronectin, indirect effects of plasmin such as activation of collagenases may be involved. ${ }^{39-41}$ Histological examination revealed no evidence of retinal damage in any plasmin treated eye. In particular, the ILM showed no change in morphology in plasmin treated or control eyes which suggests that plasmin did not degrade type IV collagen. As type IV collagen is the main structural protein of basement membranes, the absence of a proteolytic effect on collagen IV may be a major advantage of plasmin compared with dispase. It has been reported previously that dispase cleaved the vitreoretinal attachment but caused "minimal damage to the inner retina". ${ }^{42}$

At the vitreous base cortical vitreous collagen fibrils run perpendicular to the retinal surface and not parallel as at the posterior pole and the equator. ${ }^{43}{ }^{44}$ In our series there was no difference between plasmin treated eyes and controls with regard to the ultrastructure of the vitreous base, indicating that plasmin did not cleave the vitreoretinal junction by secondary activation of collagenases. 
Adult domestic pig eyes were used because of their thick posterior hyaloid and strong adherence of the vitreous cortex to the ILM. There are species related differences in the vitreoretinal junction. ${ }^{38}$ The vitreoretinal junction of pig eyes is strong, and PVD is not known to occur spontaneously in this species. The pig is therefore a difficult model for the study of vitreoretinal adhesion and separation. Any investigation of the cleaving effect of plasmin requires standardised characteristics of the ultrastructure of the vitreoretinal interface at baseline, such as an attached vitreous and an intact blood-retinal barrier. We therefore used eyes from freshly slaughtered pigs as a cheap and easily available model for this study.

Provided plasmin has a similar cleaving effect at the vitreoretinal interface of human eyes without damaging the retina or other intraocular structures, it may prove to be a viable biochemical adjunct to vitrectomy. With regard to the ability of plasmin to create a smooth retinal surface without remnants of cortical vitreous, plasmin assisted vitrectomy may not only facilitate surgical vitreoretinal separation but may also reduce the risk of subsequent cellular proliferation and fibrocellular contraction.

Supported by Hermann Wacker-Fond, Stifterverband für die Deutsche Wissenschaft (T006/01.004/99).

Proprietary interest: none.

1 Akiba J, Quiroz MA, Trempe CL. Role of posterior vitreous detachment in idiopathic macular holes. Ophthalmology detachment in 1 id

2 Akiba J, Arzabe CW, Trempe CL. Posterior vitreous detachment and neovascularization in diabetic retinopathy

3 Akiba J, Kado M, Kakehashi A, et al. Role of the vitreous in posterior segment neovascularization in central retinal vein occlusion. Ophthalmic Surg 1991;22:498-502.

4 Avunduk AM, Cetinkaya K, Kapicioglu Z, et al. The effect of posterior vitreous detachment on the prognosis of branch retinal vein occlusion. Acta Ophthalmol Scand 1997 75:441-2.

5 Hikichi T, Akiba J, Trempe CL. Effect of the vitreous on the prognosis of full-thickness idiopathic macular hole. $A m \mathcal{F}$ Ophthalmol 1993:116:273-8.

6 Hikichi T, Fujio N, Trempe CL. The vitreous in retinal arterial occlusions. Retina 1994;14:335-7.

7 Hikichi T, Trempe CL, Schepens CL. Posterior vitreous detachment as a risk factor for retinal detachment. detachment as a risk factor
Ophthalmology 1995;102:527-8.

8 Hikichi T, Yoshida A, Trempe CL. Course of vitreomacular traction syndrome. Am f Ophthalmol 1995;119:55-61.

9 Hikichi T, Konno S, Trempe CL. Role of the vitreous in central retinal vein occlusion. Retina 1995;15:29-33.

10 Jalkh AE, Trempe CL. Vitreous changes and macular disease. Bull Soc Belge Ophtalmol 1987;223(Pt 1):155-64.

11 Nasrallah FP, Jalkh AE, Van Coppenolle F, et al. The role of the vitreous in diabetic macular edema. Ophthalmology 1988;95:1335-9.

12 Schepens CL, Avila MP, Jalkh AE, et al. Role of the vitreous in cystoid macular edema. Surv Ophthalmol 1984; 28(Suppl):499-504.

13 Schepens CL. Vitreous changes in retinal detachment. Bull Soc Belge Ophtalmol 1987;223(Pt 1):85-107.

14 Sebag J. Anatomy and pathology of the vitreo-retinal interface. Eye 1992;6:541-2.

15 Trempe CL, McMeel JW, Jalkh AE. Role of the vitreous in retinal vascular disorders. Bull Soc Belge Ophtalmol 1987;223(Pt 2):1-4.

16 Gandorfer A, Messmer EM, Ulbig MW, et al. Resolution of diabetic macular edema after surgical removal of the posterior hyaloid and the inner limiting membrane. Retina 2000 20:126-33.
17 Harbour JW, Smiddy WE, Rubsamen PE, et al. Pars plana vitrectomy for chronic pseudophakic cystoid macular vitrectomy for chronic pseudophakic cyst

18 Lewis H, Abrams GW, Blumenkranz MS, et al. Vitrectomy for diabetic macular traction and edema associated with posterior hyaloidal traction. Ophthalmology 1992;99:753-9.

19 Harbour JW, Smiddy WE, Flynn HWJ, et al. Vitrectomy for diabetic macular edema associated with a thickened and taut posterior hyaloid membrane. Am $f$ Ophthalmol 1996;121:405-13.

20 Han DP, Abrams GW, Aaberg TM. Surgical excision of the attached posterior hyaloid. Arch Ophthalmol 1988;106:9981000.

21 Le Mer Y, Kroll P, Chofflet J, et al. Recherche systematique du cortex vitreen posterieur lors de la vitrectomie. Technique, complications et resultats. F Fr Ophtalmol 1994; 17:459-64.

22 Vander JF, Kleiner R. A method for induction of posterior vitreous detachment during vitrectomy [see comments]. Retina 1992;12:172-3.

23 Casaroli MR, Vilaro S. The role of fibronectin, laminin, vitronectin and their receptors on cellular adhesion in proliferative vitreoretinopathy. Invest Ophthalmol Vis Sci 1994;35: 2791-803.

24 Wiedemann P, Weller M, Heimann K. Proliferative Vitreoretinopathie: Neue Erkenntnisse in Pathophysiologie und Therapie. Klin Monatsbl Augenheilkd 1990;197:35561.

25 Sebag J. Pharmacologic vitreolysis. Retina 1998;18:1-3.

26 Kohno T, Sorgente N, Goodnight R, et al. Alterations in the distribution of fibronectin and laminin in the diabetic human eye. Invest Ophthalmol Vis Sci 1987;28:515-21.

27 Kohno T, Sorgente N, Ishibashi T, et al. Immunofluorescent studies of fibronectin and laminin in the human eye. Invest Ophthalmol Vis Sci 1987;28:506-14.

28 Liotta LA, Goldfarb RH, Brundage R, et al. Effect of plasminogen activator (urokinase), plasmin, and thrombin on glycoprotein and collagenous components of basement membrane. Cancer Res 1981;41:4629-36.

29 Papp B, Kovacs T, Lerant I, et al. Conditions of formation of the heparin-fibronectin-collagen complex and the effect of plasmin. Biochim Biophys Acta 1987;925:241-7 [erratum, Biochim Biophys Acta 1988;964:293].

30 Akiba J, Ueno N, Chakrabarti B. Molecular mechanisms of posterior vitreous detachment. Graefes Arch Clin Exp Ophthalmol 1993;231:408-12.

31 Hikichi T, Yanagiya N, Kado M, et al. Posterior vitreous detachment induced by injection of plasmin and sulfur hexafluoride in the rabbit vitreous. Retina 1999;19:55-8.

32 Verstraeten TC, Chapman C, Hartzer M, et al. Pharmacologic induction of posterior vitreous detachment in the rabbit. Arch Ophthalmol 1993;111:849-54.

33 Hesse L, Nebeling B, Schroeder B, et al. Induction of posterior vitreous detachment in rabbits by intravitreal injection of tissue plasminogen activator following cryopexy. Exp Eye of tissue plasminog
Res 2000;70:31-9.

34 Garcia-Layana A, Pastor JC, Saornil MA, et al. Porcine model of proliferative vitreoretinopathy with platelets. Curr Eye Res 1997;16:556-63

35 Hines J, Vinores SA, Campochiaro PA. Evolution of morphologic changes after intravitreous injection of gentamicin. Curr Eye Res 1993;12:521-9.

36 Prince JH, Diesem CD, Eglitis I, et al. The pig. In: Anatomy and histology of the eye and orbit in domestic animals. Springfield, IL: Charles C Thomas, 1960:210-33.

37 Bloodworth JMB, Gutgesell HP, Engermann RL. Retinal vasculature of the pig: light and electron microscopic studies. Exp Eye Res 1965;4:174-8.

38 Matsumoto B, Blanks JC, Ryan SJ. Topographic variations in the rabbit and primate internal limiting membrane. Invest Ophthalmol Vis Sci 1984;25:71-82.

39 Dano K, Andreasen PA, Grondahl-Hansen J, et al. Plasminogen activators, tissue degradation, and cancer. Plasminogen activators, tissue degr Res 1985;44:139-266.

40 Mainardi CL, Hasty DL, Seyer JM, et al. Specific cleavage of human type III collagen by human polymorphonuclear eukocyte elastase. F Biol Chem 1980;255:12006-10.

41 Mainardi CL, Dixit SN, Kang AH. Degradation of type IV (basement membrane) collagen by a proteinase isolated rom human polymorphonuclear leukocyte granules. $\mathcal{F}$ Biol Chem 1980;255:5435-41

42 Tezel TH, Del Priore LV, Kaplan HJ. Posterior vitreous detachment with dispase [see comments]. Retina 1998;18: $7-15$.

43 Gartner J. Electron-microscopic study on the fibrillar network and fibrocyte-collagen interactions in the vitreous cortex at the ora serrata of human eyes with special regard to the role of disintegrating cells. Exp Eye Res 1986;42:2133 .

44 Sebag J, Balazs EA. Morphology and ultrastructure of human vitreous fibers. Invest Ophthalmol Vis Sci 1989;30: 1867-71. 